Article

\title{
Mie-Metamaterials-Based Thermal Emitter for Near-Field Thermophotovoltaic Systems
}

\author{
Alok Ghanekar ${ }^{1}$ (D), Yanpei Tian ${ }^{1}$, Sinong Zhang ${ }^{2}$, Yali Cui ${ }^{2,3}$ and Yi Zheng ${ }^{1, *}$ \\ 1 Department of Mechanical, Industrial and Systems Engineering, University of Rhode Island, \\ Kingston, RI 02881, USA; alokg@my.uri.edu (A.G.); yanpei_tian@my.uri.edu (Y.T.) \\ 2 College of Life Sciences, Northwest University, Xi'an 710069, China; \\ zhangsinong@stumail.nwu.edu.cn (S.Z.); yalicui@nwu.edu.cn (Y.C.) \\ 3 National Engineering Research Center for Miniaturized Detection Systems, Northwest University, \\ Xi'an 710069, China \\ * Correspondence: zheng@uri.edu; Tel.: +1-401-874-5184
}

Received: 28 June 2017; Accepted: 29 July 2017; Published: 31 July 2017

\begin{abstract}
In this work, we theoretically analyze the performance characteristics of a near-field thermophotovoltaic system consisting a Mie-metamaterial emitter and GaSb-based photovoltaic cell at separations less than the thermal wavelength. The emitter consists of a tungsten nanoparticle-embedded thin film of $\mathrm{SiO}_{2}$ deposited on bulk tungsten. Numerical results presented here are obtained using formulae derived from dyadic Green's function formalism and Maxwell-Garnett-Mie theory. We show that via the inclusion of tungsten nanoparticles, the thin layer of $\mathrm{SiO}_{2}$ acts like an effective medium that enhances selective radiative heat transfer for the photons above the band gap of $\mathrm{GaSb}$. We analyze thermophotovoltaic (TPV) performance for various volume fractions of tungsten nanoparticles and thicknesses of $\mathrm{SiO}_{2}$.
\end{abstract}

Keywords: near-field thermal radiation; thermophotovoltaics; Mie-metamaterials; effective medium theory

\section{Introduction}

Thermophotovoltaics (TPVs) have been the focus of several works as an alternative to power generation technologies and a technology for waste heat recovery systems [1-3]. A typical TPV system consists of a high-temperature ( $1500 \mathrm{~K})$ thermal emitter and a photovoltaic (PV) cell that converts the energy of incident photons into electricity. While ideal TPV systems convert radiative energy into electricity at an efficiency of the Carnot engine, practical TPV systems suffer from mismatch between the emission spectra of the emitter and absorption spectra of PV cell [4-8]. Several studies have investigated the use of metamaterials [9-11], surface gratings and photonics crystals [12-17], and complex surface patterns [18] to improve efficiency of TPV systems. In the last few years, it was demonstrated that near-field thermal radiation has a great potential in improving TPV systems [19-24]. Several theoretical studies were focused on exploiting near-field coupling of surface waves between emitter and PV cell at nanometer separation to improve the efficiency. It is challenging to develop materials that can withstand high temperatures as well as allow an enhanced coupling of surface waves for the energies above the band gap of the PV cell. One-dimensional gratings, hyperbolic metamaterials, and photonic crystals have shown a great potential in TPV applications. Dielectric mixtures and nanoparticle-embedded thin films can also be utilized to tune near-field thermal radiation [25]. It has been previously demonstrated that Mie-metamaterials or Mie-resonance metamaterials that utilize Mie resonances of inclusions into host materials can be used for spectral tuning of near-field thermal radiation. Authors demonstrated the possible use of tungsten nanoparticle-embedded thin film of $\mathrm{SiO}_{2}$ to achieve a selective thermal emitter for a far-field TPV system [26]. The effect of nanoparticle 
inclusions into host material on near-field radiative heat transfer has been investigated in several theoretical studies [27-29]. Stemming from earlier work, we demonstrate the use of a Mie-metamaterial thermal emitter that consists of tungsten nanoparticles embedded in a thin film of $\mathrm{SiO}_{2}$ deposited on a thick layer of tungsten for a near-field TPV system. We also explore possible alternative of W nanoparticles by refractive materials such as titanium nitride (TiN), tantalum (Ta), and molybdenum (Mo). We investigate the performance of such a TPV system for various configurations. While many recent works have dealt with gratings, photonic crystals, and other metamaterials, this is the first time nanoparticle-embedded thin films have been investigated for a near-field TPV system.

The configuration of the near-field TPV system considered in the present study is shown in Figure 1. The emitter side is a Mie-metamaterial consisting of tungsten nanoparticles embedded in $\mathrm{SiO}_{2}$ thin film on the top of a tungsten layer. The radius of the nanoparticles was fixed at $20 \mathrm{~nm}$. The volume fraction and thickness of the $\mathrm{SiO}_{2}$ layer can be varied to investigate behaviour of the system. The layer of tungsten blocks radiation from the substrate, making the emitter essentially opaque. The PV cell considered here is $\mathrm{GaSb}$ (which has a bandgap of $0.726 \mathrm{eV}$ ), whose properties can be found in Reference [30]. Calculations reported in this work are for emitter temperature of $1500 \mathrm{~K}$, while the PV cell is assumed to be at $300 \mathrm{~K}$. The separation between the emitter and PV cell is comparable to or less than the thermal wavelength at $1500 \mathrm{~K}\left(\lambda_{t h}=1.93 \mu \mathrm{m}\right)$.

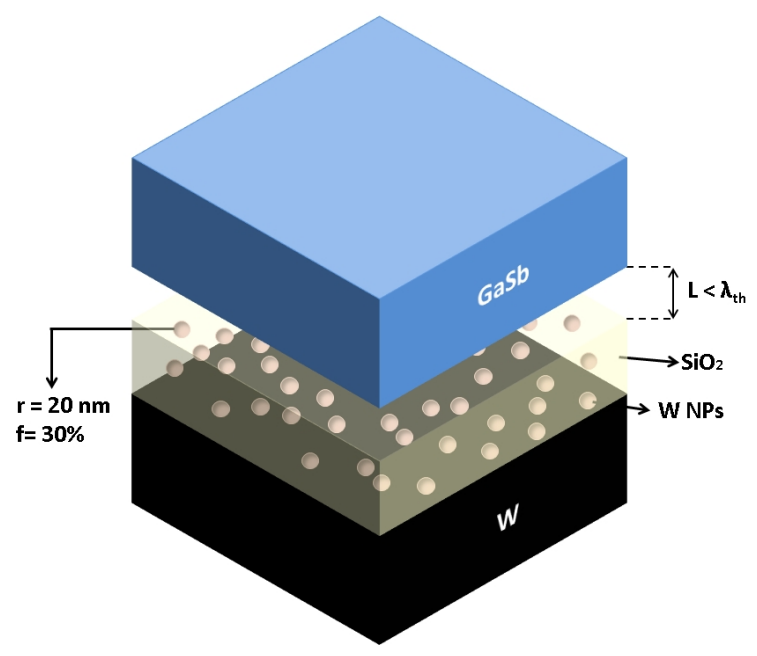

Figure 1. Schematic of near-field thermophotovoltaic system consisting of the proposed thermal emitter and GaSb-based PV cell at separation less than the thermal wavelength.

While we mostly concern ourselves with the theoretical side of the proposed thermal emitter, there are various ways to fabricate nanoparticle-embedded thin films. For example, it has been demonstrated that a stack of metallic nanoparticle arrays and $\mathrm{SiO}_{2}$ arrays can be fabricated [31]. Alternatively, as suggested by [32], core-shell nanoparticle arrays can be fabricated using tungsten core and $\mathrm{SiO}_{2} \mathrm{shell}_{\text {. }}$ This is followed by sputtering or chemical vapor deposition of $\mathrm{SiO}_{2}$. W Nanoparticles-SiO 2 composites can also be fabricated using sputtering deposition process [33-35]. A mixture of $\mathrm{W}$ and $\mathrm{SiO}_{2}$ powders can be prepared using PVDF (polyvinylidene fluoride) and sintering/pressing onto a sputtering cooling plate. $\mathrm{W}-\mathrm{SiO}_{2}$ composites can be fabricated by RF sputtering of $\mathrm{W}$ followed by sputtering of $\mathrm{W}-\mathrm{SiO}_{2}$ powder. During the sputtering process, tungsten nanoparticles would form.

\section{Results and Discussion}

The refractive indices of plain $\mathrm{SiO}_{2}$ and that of $\mathrm{SiO}_{2}$ mixed with $30 \%$ nanoparticles of tungsten are shown in Figure 2. The effect of nanoparticle inclusions can be observed. $\mathrm{SiO}_{2}$ has a near-constant value of refractive index $(n)$ and a negligible extinction coefficient $(\kappa)$ in the spectral range of our 
interest. The dielectric mixture of $\mathrm{SiO}_{2}$ and tungsten displayed an overall increased effective refractive index and a higher absorption coefficient for wavelengths shorter than $2.5 \mu \mathrm{m}$.

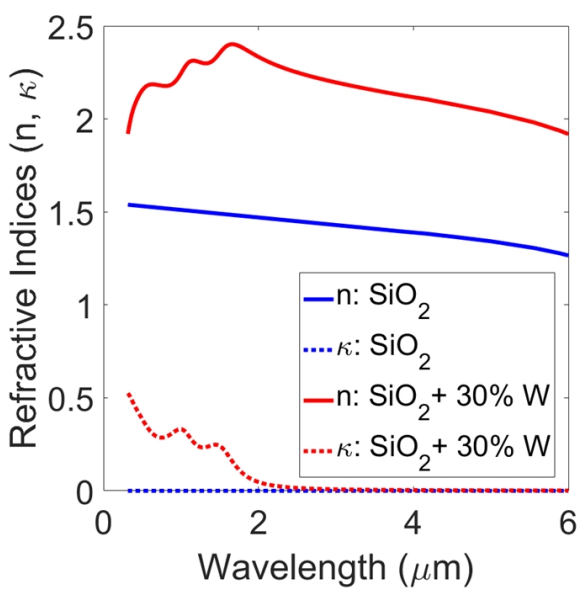

Figure 2. Real (n) and imaginary ( $\kappa$ ) parts of refractive indices of pure $\mathrm{SiO}_{2}$ and $\mathrm{SiO}_{2}$ with $30 \%$ tungsten nanoparticles.

It is crucial to reduce the spectral energy below the band-gap of the PV cell in order to improve the overall thermal efficiency of the TPV system. The coupling of surface waves dictates whether radiative transfer would be enhanced or suppressed. The goal is to enhance radiative transfer above the bandgap without significantly enhancing radiation below the bandgap of the PV cell. In order to assess the impact of nanoparticle inclusions, we investigate the spectral heat flux of the proposed TPV system for various configurations. The spectral heat flux across the proposed thermal emitter at a separation of $L=100 \mathrm{~nm}$ for various compositions is plotted in Figure 3. The emitter with a $0.3 \mu \mathrm{m}$ layer of $\mathrm{SiO}_{2}$ on tungsten had lower heat flux across the spectrum when compared to plain tungsten, as seen in Figure 3a. However, upon the inclusion of tungsten nanoparticles, spectral heat flux increased and was more selective towards shorter wavelengths $(\lambda<2 \mu \mathrm{m})$. While spectral heat flux showed an increase over the entire range, it was more prominent for energies above the band-gap, leading to a lesser fraction of energy lost. An increased absorption coefficient in the shorter wavelengths can be accounted for by enhanced coupling of surface modes in that range. To demonstrate the effect of reducing bulk layer to thin layer, Figure $3 \mathrm{~b}$ illustrates spectral responses for various thicknesses of $\mathrm{SiO}_{2}$ layer and a fixed nanoparticle volume fraction of $30 \%$. While a bulk layer of $\mathrm{SiO}_{2}$ mixed with tungsten nanoparticle showed broadband heat transfer, it can be clearly seen that thinner layers of $\mathrm{SiO}_{2}$ yielded more selective spectral response while maximum spectral heat flux remained relatively constant. It can be observed that radiation at longer wavelengths was more sensitive to layer thickness-such a configuration is more desirable for minimizing losses due to long-wavelength photons. In principle, it is possible to tune the near-field thermal radiation by changing the volume fraction of nanoparticles and thickness of the $\mathrm{SiO}_{2}$ layer to achieve optimal configuration for a given operating temperature and separation.

We now investigate the power output of the GaSb PV cell as a result of near-field radiative heat transfer at a separation of $100 \mathrm{~nm}$. To assess the overall performance of the TPV system, we model the PV cell as discussed in Section 3. We calculate spectral density of output power along with the total output power. Figure 4 shows calculated spectral density of output power from the PV cell for the emitter with a pure $\mathrm{SiO}_{2}$ layer of $0.3 \mu \mathrm{m}$ thickness on tungsten and $\mathrm{SiO}_{2}$ layer with $30 \%$ tungsten nanoparticles. The emitter with a tungsten nanoparticles-embedded thin film of $\mathrm{SiO}_{2}$ displayed an enhanced output power contribution above the bandgap of the GaSb PV cell when compared with the emitter with pure $\mathrm{SiO}_{2}$ layer. For comparison, the spectral heat flux across the interface of the same configurations are also shown. The total heat flux and total power output of the PV cell of these 
configurations are plotted against separation up to $10 \mathrm{~nm}$ in Figure 5 . For gaps larger than $1 \mu \mathrm{m}$ (far-field), heat flux (and consequently power power and system efficiencies) were independent of the distance. For separations less than $1 \mu \mathrm{m}$ (near-field), the overall heat flux increased due to the presence of evanescent waves. Consequently, the output power also rose monotonically as separation between the emitter and PV cell was reduced. The inset in Figure 5 shows TPV system efficiency against distance for the same setups. While the trend in efficiencies is not as monotonous, for separations smaller than $100 \mathrm{~nm}$, the emitter with tungsten nanoparticles showed higher thermal efficiency than the one with pure $\mathrm{SiO}_{2}$. This is supported by earlier results that show the increased selectivity at shorter wavelengths leading to lower losses. Interestingly, the emitter with pure $\mathrm{SiO}_{2}$ had higher efficiency in the far-field. Nevertheless, such a configuration had lower output power. The oscillatory behaviour of efficiency has been observed before, and can be attributed to vacuum gap behaving like a waveguide [4]. We would like to emphasize that in order to further improve the performance of the TPV system, the materials chosen are not necessarily optimal. For example, PV cells with lower band gap (e.g., InGaAs and InGaSb or quaternary alloys like InGaAsSb) can be used. This can allow the use of lower emitter temperature or higher efficiencies and output power at the same operating temperatures. Alternatively, other materials, such as $\mathrm{Al}_{2} \mathrm{O}_{3}$ (in place of $\mathrm{SiO}_{2}$ ) and platinum, molybdenum, tantalum, and titanium nitride (in place of $\mathrm{W}$ ) can be investigated for thermal emitter structure. We show a possible replacement of $\mathrm{W}$ by refractory materials such as titanium nitride (TiN), molybdenum (Mo), and tantalum (Ta) using our calculations in Figure 6. Spectral heat flux between the PV cell and emitter with $\mathrm{SiO}_{2}$ layer of $0.3 \mu \mathrm{m}$ thickness and 30\% nanoparticles at a separation of $100 \mathrm{~nm}$ is shown. For comparison, heat flux with tungsten nanoparticles is shown. Corresponding values of total heat flux for W, TiN, Mo, and Ta nanoparticles were $3.72 \times 10^{5}, 3.81 \times 10^{5}, 2.68 \times 10^{5}$ and $2.57 \times 10^{5} \mathrm{~W} / \mathrm{m}^{2}$, respectively. From Figure 6 it appears that emitter with TiN and W nanoparticles displayed higher heat flux for this particular configuration. The emitter with tungsten nanoparticles showed better selectivity than TiN. Various combinations of material type and dimensions can be investigated to tune the emission spectra.
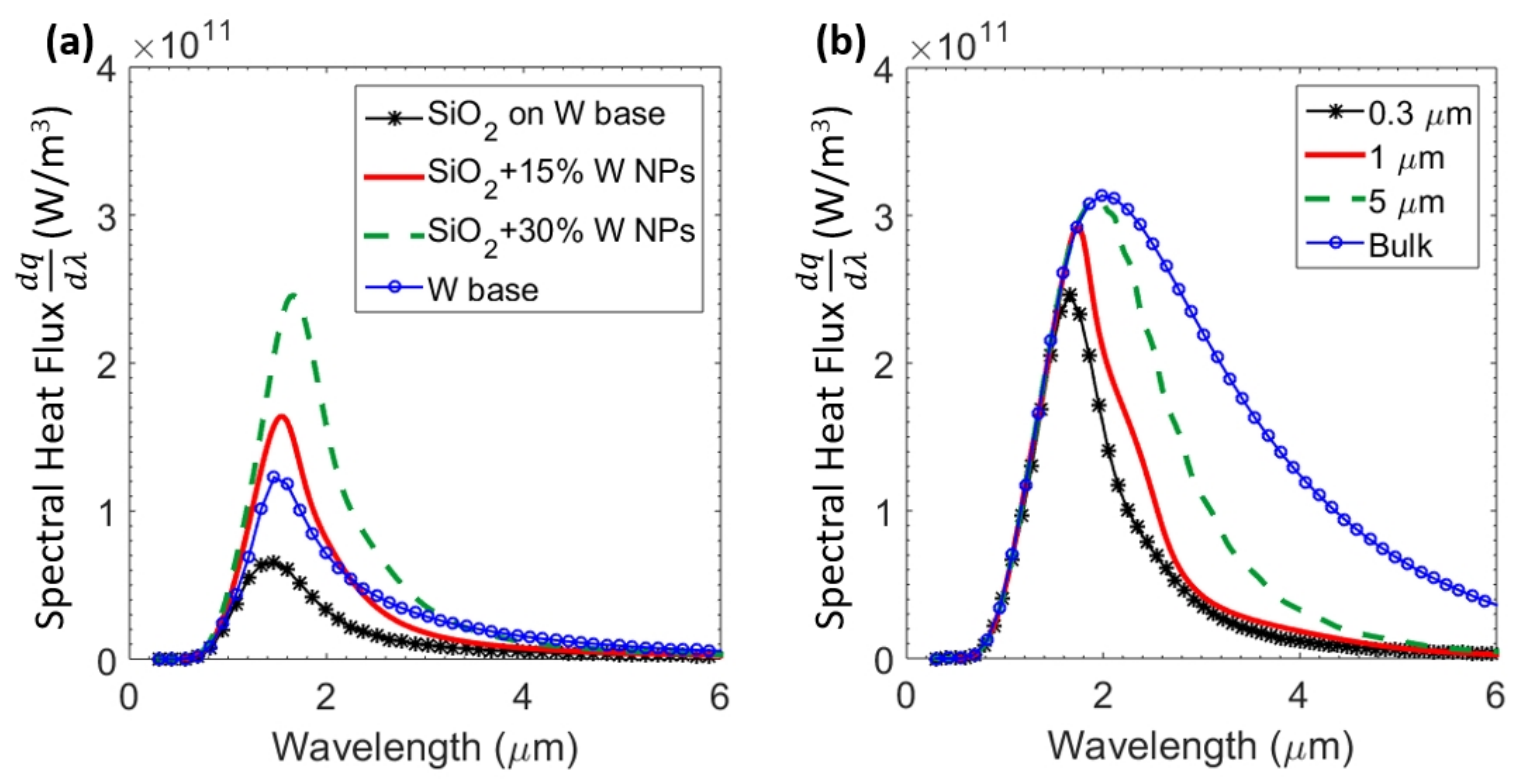

Figure 3. Spectral heat flux across the proposed emitter and the GaSb photovoltaic (PV) cell at a separation of $\mathrm{L}=100 \mathrm{~nm}$ for (a) various volume fractions of $\mathrm{W}$ nanoparticles- $0 \%, 15 \%$ and $30 \%$ - compared to bulk W emitter; (b) Various thicknesses of $\mathrm{SiO}_{2}$ layer- $0.3 \mu \mathrm{m}, 1 \mu \mathrm{m}, 5 \mu \mathrm{m}$, and bulk respectively. 


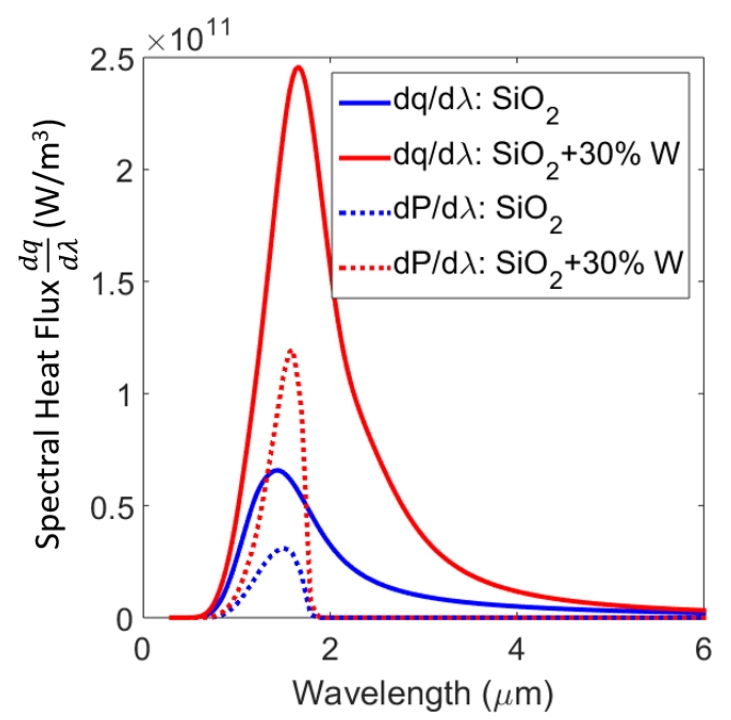

Figure 4. Predicted spectral density of output power (dashed lines) from GaSb PV cell for emitter with pure $\mathrm{SiO}_{2}$ thin film and $\mathrm{SiO}_{2}$ with $30 \%$ of $\mathrm{W}$ nanoparticles for a separation of $100 \mathrm{~nm}$ compared with corresponding spectral heat fluxes (solid lines).

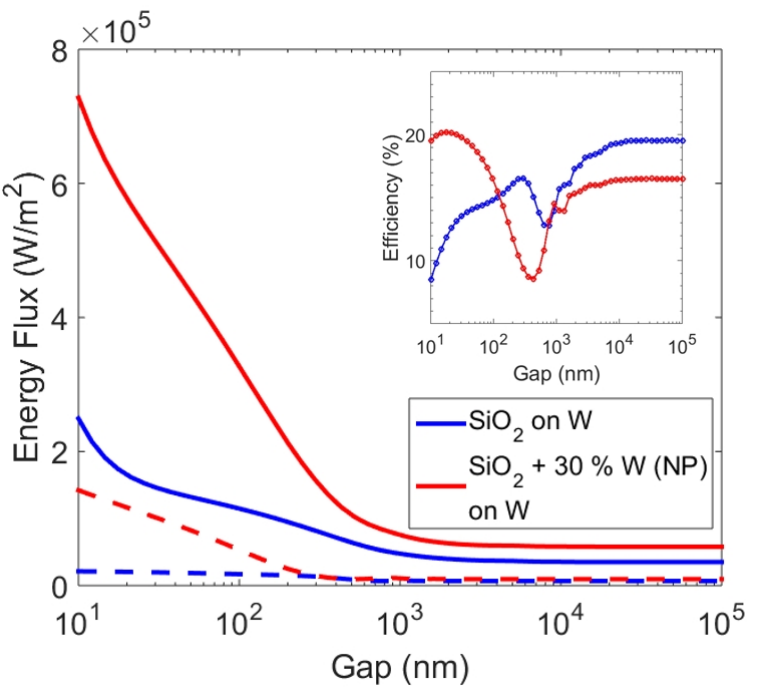

Figure 5. Total heat flux (solid lines) and output power (dashed lines) of PV cell as a function of separation between the emitter and PV cell for an emitter of pure $\mathrm{SiO}_{2}$ film of $0.5 \mu \mathrm{m}$ and $\mathrm{SiO}_{2}$ films with $\mathrm{W}$ nanoparticles. Inset shows overall efficiency of the corresponding thermophotovoltaic (TPV) systems plotted as a function of separation. NP: nanoparticle.

Overall, we have conducted the first numerical investigation of a near-field thermophotovoltaic system that uses a Mie-metamaterial-based thermal emitter and a PV cell at a separation less than the thermal wavelength. We have theoretically demonstrated an enhanced wavelength-selective thermal emitter for near-field thermophotovoltaic system using a Mie-resonance metamaterial. The thermal emitter consists of tungsten nanoparticle-embedded thin film of $\mathrm{SiO}_{2}$ deposited on a thick tungsten substrate. We analyzed the performance of such a TPV device for various cases. We studied the effect of volume fraction, layer thickness of $\mathrm{SiO}_{2}$, and separation between emitter and PV cell. The embedded tungsten nanoparticles in the thin film can alter the refractive index of the film and allow spectral control of near-field radiative transfer across the emitter and the PV cell. We evaluated the energy conversion efficiency of the proposed near-field thermophotovoltaic system. The results show that the structure of the Mie-metamaterial thermal emitter can significantly improve the efficiency of 
the thermophotovoltaic system. Improvement in spectral selectivity as well as overall heat transfer can be attributed to increased power output and efficiency. We showed that by changing the volume fraction of nanoparticles and the thickness of the $\mathrm{SiO}_{2}$ layer, it is possible to tune the near-field thermal radiation to obtain enhanced output power and high thermal efficiency. The materials considered can withstand high temperatures and are suitable for thermal emitters.

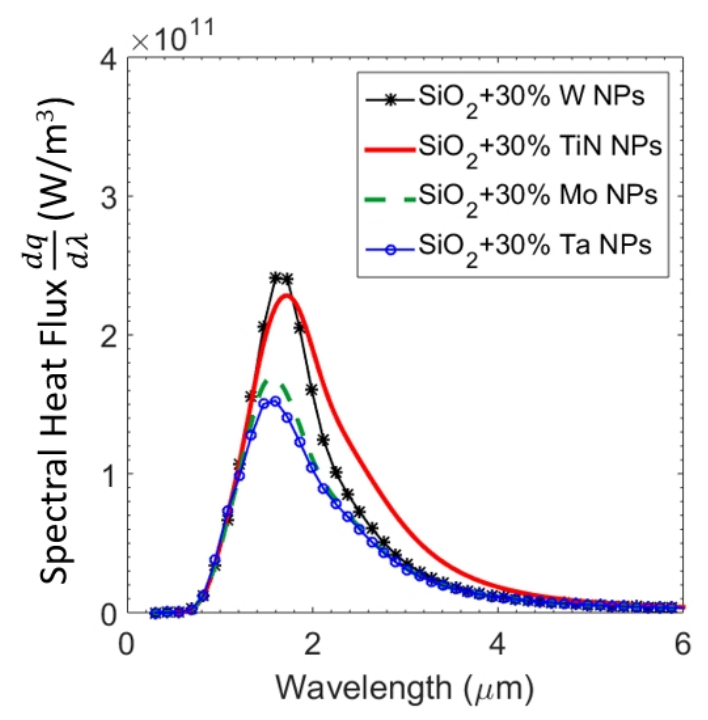

Figure 6. Spectral heat flux across the emitter consisting of nanoparticles of alternative materials and the GaSb PV cell at a separation of $L=100 \mathrm{~nm}$ for a nanoparticles volume fraction of $30 \%$.

\section{Materials and Methods}

The expression of radiative transfer between closely-spaced bodies can be derived using dyadic Green's function approach [36], and is given by

$$
q_{1 \rightarrow 2}\left(T_{1}, T_{2}, L\right)=\int_{0}^{\infty} \frac{d \omega}{2 \pi}\left[\Theta\left(\omega, T_{1}\right)-\Theta\left(\omega, T_{2}\right)\right] T_{1 \rightarrow 2}(\omega),
$$

where $\Theta(\omega, T)=(\hbar \omega / 2) \operatorname{coth}\left(\hbar \omega / 2 k_{B} T\right)$ is the energy of a harmonic oscillator at frequency $\omega$, temperature $T, \hbar$ is the reduced Planck constant, and $k_{B}$ is the Boltzmann constant. The function $T_{1 \rightarrow 2}(\omega)$ corresponds to the spectral transmissivity in radiative transfer between media 1 and 2 separated by distance $L$ and is expressed as [36]

$$
\begin{aligned}
T_{1 \rightarrow 2}(\omega) & =\int_{0}^{\omega / c} \frac{k_{\rho} d k_{\rho}}{2 \pi} \sum_{\mu=s, p} \frac{\left(1-\left|\widetilde{R}_{h 1}^{(\mu)}\right|^{2}\right)\left(1-\left|\widetilde{R}_{h 2}^{(\mu)}\right|^{2}\right)}{\left|1-\widetilde{R}_{h 1}^{(\mu)} \widetilde{R}_{h 2}^{(\mu)} e^{2 j k_{h z} L}\right|^{2}} \\
& +\int_{\omega / c}^{\infty} \frac{k_{\rho} d k_{\rho}}{2 \pi} \sum_{\mu=s, p} \frac{4 \Im\left(\widetilde{R}_{h 1}^{(\mu)}\right) \Im\left(\widetilde{R}_{h 2}^{(\mu)}\right) e^{-2\left|k_{h z}\right| L}}{\mid 1-\widetilde{R}_{h 1}^{(\mu)} \widetilde{R}_{h 2}^{(\mu)} e^{-\left.2\left|k_{h z}\right| L\right|^{2}}},
\end{aligned}
$$

where $\widetilde{R}_{h 1}^{(\mu)}$ and $\widetilde{R}_{h 2}^{(\mu)}$ are polarized effective reflection coefficients of the two half spaces (calculated in the absence of other half space), and $k_{h z}$ is the $z$-component of wavevector in vacuum. The first term in Equation (2) corresponds to propagating waves, while the second term describes the thermal transport due to evanescent waves, and its contribution is significant only for small values of gap $L$. For a structure having $N$-layer media having $(N-1)$ interfaces, the expression for the generalized reflection coefficient at the interface between region $i$ and region $i+1$ is given by [37]: 


$$
\widetilde{R}_{i, i+1}^{(\mu)}=\frac{R_{i, i+1}^{(\mu)}+\widetilde{R}_{i+1, i+2}^{(\mu)} e^{2 j k_{i+1, z}\left(d_{i+1}-d_{i}\right)}}{1+R_{i, i+1}^{(\mu)} \widetilde{R}_{i+1, i+2}^{(\mu)} e^{2 j k_{i+1, z}\left(d_{i+1}-d_{i}\right)}},
$$

where $R_{i, i+1}^{(\mu)}$ is the Fresnel reflection coefficient at the interface between layers $i$ and $i+1$, and $\widetilde{R}_{i+1, i+2}^{(\mu)}$ is the generalized reflection coefficient at the interface between layers $i+1$ and $i+2, \mu=s$ (or $p$ ) refers to transverse electric (or magnetic) polarization, $z=-d_{i}$ is the location of the $i$ th interface. $k_{i, z}=\sqrt{\varepsilon_{i}(\omega) \omega^{2} / c^{2}-k_{\rho}^{2}}$ is the normal $z$-component of the wave vector in medium $i$, wherein $\varepsilon_{i}(\omega)$ is the relative permittivity of the medium $i$ as a function of angular frequency $\omega, c$ is the speed of light in vacuum, and $k_{\rho}$ is the magnitude of the in-plane wave vector. With $\widetilde{R}_{N, N+1}^{(\mu)}=0$, the above equation provides a recursive relation to calculate the reflection coefficients $\widetilde{R}_{i, i+1}^{(\mu)}$ in all regions. To calculate the effective dielectric function of the Mie-metamaterial, we use the Clausius-Mossotti equation [38,39].

$$
\varepsilon_{e f f}=\varepsilon_{m}\left(\frac{r^{3}+2 \alpha_{r} f}{r^{3}-\alpha_{r} f}\right)
$$

where $\varepsilon_{m}$ is the dielectric function of the matrix, $\alpha_{r}$ is the electric dipole polarizability, and $r$ and $f$ are the radius and volume fraction of nanoparticles, respectively. To consider the size effects of nanoparticle inclusions, we use the Maxwell-Garnett formula, which employs the expression for electric dipole polarizability using Mie theory [40], $\alpha_{r}=3 j c^{3} a_{1, r} / 2 \omega^{3} \varepsilon_{m}^{3 / 2}$, where $a_{1, r}$ is the first electric Mie coefficient given by

$$
a_{1, r}=\frac{\sqrt{\varepsilon_{n p}} \psi_{1}\left(x_{n p}\right) \psi_{1}^{\prime}\left(x_{m}\right)-\sqrt{\varepsilon_{m}} \psi_{1}\left(x_{m}\right) \psi_{1}^{\prime}\left(x_{n p}\right)}{\sqrt{\varepsilon_{n p}} \psi_{1}\left(x_{n p}\right) \xi_{1}^{\prime}\left(x_{m}\right)-\sqrt{\varepsilon_{m}} \xi_{1}\left(x_{m}\right) \psi_{1}^{\prime}\left(x_{n p}\right)},
$$

where $\psi_{1}$ and $\xi_{1}$ are Riccati-Bessel functions of the first order given by $\psi_{1}(x)=x j_{1}(x)$ and $\xi_{1}(x)=x h_{1}^{(1)}(x)$ where $j_{1}$ and $h_{1}^{(1)}$ are first-order spherical Bessel functions and spherical Hankel functions of the first kind, respectively. Here, " ' " indicates the first derivative. $x_{m}=\omega r \sqrt{\varepsilon_{m}} / c$ and $x_{n p}=\omega r \sqrt{\varepsilon_{n p}} / c$ are the size parameters of the matrix and the nanoparticles, respectively; $\varepsilon_{n p}$ is the dielectric function of nanoparticles. It is worth mentioning that Maxwell-Garnett-Mie theory is applicable when the average distance between inclusions is much smaller than the wavelength of interest [41]. This criteria is satisfied in the calculations presented. Since nanoparticle diameter (40 nm) is much smaller than the thickness of the thin film $(0.3 \mu \mathrm{m})$ considered, effective medium theory holds true for the calculations presented in this study. Dielectric functions of the materials $\left(\mathrm{SiO}_{2}\right.$ and $\left.\mathrm{W}\right)$ considered in this paper are taken from literature [42,43]. Having very low temperature coefficients, room temperature values of dielectric function are used for $\mathrm{SiO}_{2}$ [44]. Dielectric properties of tungsten were also assumed to be unchanged, as the operating temperature is much less than the melting point.

Near-field thermal radiation and charge transport in PV cell can be theoretically modelled by accounting for charge density distribution due to the number of photons absorbed at different cell depths [22]. Due to the limitations of the present study, we employ a simplistic model to calculate the output power of a PV cell. We assume that quantum efficiency of a PV cell in the near-field would be the same as that in the far-field. Therefore, short circuit current can be calculated as [22]

$$
I_{S C}=\int_{E_{g} / \hbar}^{\infty} \frac{e}{\hbar \omega} \cdot E Q E_{G a S b}(\omega) \cdot \frac{d q}{d \omega} d \omega,
$$

where $E_{g}$ is the bandgap of GaSb cell, $E Q E_{G a S b}$ is external quantum efficiency, $d q / d \omega$ is spectral heat flux, and $e$ is the electronic charge. Dark current can be calculated by

$$
I_{0}=\frac{e D_{n} n_{i}^{2}}{L_{n} N_{n}}+\frac{e D_{p} n_{i}^{2}}{L_{p} N_{p}}
$$


where $D_{n}$ and $D_{p}$ are diffusivities of electrons and holes, respectively, $n_{i}$ is the intrinsic carrier concentration, $N_{n}$ and $N_{p}$ are concentrations of electrons and holes, respectively, while diffusion lengths $L_{n}$ and $L_{p}$ can be calculated in terms of diffusivity and recombination lifetime $\tau$ using

$$
L_{x}=\sqrt{D \cdot \tau}
$$

Total recombination lifetime is calculated by

$$
1 / t_{\text {tot }}=1 / \tau_{R}+1 / \tau_{S H R}+1 / \tau_{A u}
$$

where $\tau_{R}, \tau_{S H R}$, and $\tau_{A u}$ are radiative recombination, Shockley-Hall recombination, and Augar recombination, respectively [45]. Open circuit voltage is calculated using

$$
V_{O C}=\left(k_{B} T_{P V} / e\right) \ln \left(I_{S C} / I_{0}+1\right) .
$$

Output power of the PV cell is given by

$$
P_{\text {out }}=I_{S C} V_{O C}(1-1 / z)[1-\ln (z) / z],
$$

where $z=\ln \left(I_{S C} / I_{0}\right)$ and efficiency of the TPV system is given by

$$
\eta=P_{\text {out }} / Q_{\text {rad }} \text {. }
$$

For our calculations, intrinsic carrier concentration was assumed to be $4.3 \times 10^{12} \mathrm{~cm}^{-3}$. Carrier concentration of electrons and holes were assumed to be equal to $N_{n}=N_{p}=10^{17} \mathrm{~cm}^{-3}$. The recombination lifetimes are taken to be $\tau_{R}=40 \mathrm{~ns}, \tau_{S H R}=10 \mathrm{~ns}$, and $\tau_{A u}=20 \mu \mathrm{s}$. Carrier diffusivities are $D_{n}=129 \mathrm{~cm}^{2} / \mathrm{s}$ and $D_{p}=39 \mathrm{~cm}^{2} / \mathrm{s}$ for electrons and holes, respectively.

Acknowledgments: This project was supported in part by an Institutional Development Award (IDeA) Network for Biomedical Research Excellence from the National Institute of General Medical Sciences of the National Institutes of Health under grant number P20GM103430, Rhode Island STAC Research Grant number AWD05085, and Rhode Island Foundation Research Grant number 20164342.

Author Contributions: A.G. did the calculations and wrote the paper. Y.T. contributed to analysis of the results and writing the paper. S.Z. and Y.C. contributed to discussion of the results and writing the paper. Y.Z. supervised the project. All authors discussed the results and commented on the manuscript.

Conflicts of Interest: The authors declare no conflict of interest.

\section{References}

1. Basu, S.; Chen, Y.; Zhang, Z. Microscale radiation in thermophotovoltaic devices-a review. Int. J. Energy Res. 2007, 31, 689-716.

2. Bosi, M.; Ferrari, C.; Melino, F.; Pinelli, M.; Spina, P.; Venturini, M. Thermophotovoltaic generation: A state of the art review. In Proceedings of the 25th International Conference on Efficiency, Cost, Optimization and Simulation of Energy Conversion Systems and Processes, Perugia, Italy, 26-29 June 2012.

3. Coutts, T. A review of progress in thermophotovoltaic generation of electricity. Renew. Sustain. Energy Rev. 1999, 3, 77-184.

4. Tong, J.K.; Hsu, W.C.; Huang, Y.; Boriskina, S.V.; Chen, G. Thin-film 'thermal well'emitters and absorbers for high-efficiency thermophotovoltaics. Sci. Rep. 2015, 5, 10661.

5. Harder, N.P.; Würfel, P. Theoretical limits of thermophotovoltaic solar energy conversion. Semicond. Sci. Technol. 2003, 18, S151.

6. Rephaeli, E.; Fan, S. Absorber and emitter for solar thermo-photovoltaic systems to achieve efficiency exceeding the Shockley-Queisser limit. Opt. Express 2009, 17, 15145-15159.

7. Wurfel, P.; Ruppel, W. Upper limit of thermophotovoltaic solar-energy conversion. IEEE Trans. Electron Devices 1980, 27, 745-750. 
8. Bermel, P.; Ghebrebrhan, M.; Chan, W.; Yeng, Y.X.; Araghchini, M.; Hamam, R.; Marton, C.H.; Jensen, K.F.; Soljačić, M.; Joannopoulos, J.D.; et al. Design and global optimization of high-efficiency thermophotovoltaic systems. Opt. Express 2010, 18, A314-A334.

9. Liu, X.; Tyler, T.; Starr, T.; Starr, A.F.; Jokerst, N.M.; Padilla, W.J. Taming the blackbody with infrared metamaterials as selective thermal emitters. Phys. Rev. Lett. 2011, 107, 045901.

10. Wu, C.; Neuner , B., III; John, J.; Milder, A.; Zollars, B.; Savoy, S.; Shvets, G. Metamaterial-based integrated plasmonic absorber/emitter for solar thermo-photovoltaic systems. J. Opt. 2012, 14, 024005.

11. Molesky, S.; Dewalt, C.J.; Jacob, Z. High temperature epsilon-near-zero and epsilon-near-pole metamaterial emitters for thermophotovoltaics. Opt. Express 2013, 21, A96-A110.

12. Nagpal, P.; Han, S.E.; Stein, A.; Norris, D.J. Efficient low-temperature thermophotovoltaic emitters from metallic photonic crystals. Nano Lett. 2008, 8, 3238-3243.

13. Arpin, K.A.; Losego, M.D.; Cloud, A.N.; Ning, H.; Mallek, J.; Sergeant, N.P.; Zhu, L.; Yu, Z.; Kalanyan, B.; Parsons, G.N.; et al. Three-dimensional self-assembled photonic crystals with high temperature stability for thermal emission modification. Nat. Commun. 2013, 4, doi:10.1038/ncomms3630.

14. Heinzel, A.; Boerner, V.; Gombert, A.; Bläsi, B.; Wittwer, V.; Luther, J. Radiation filters and emitters for the NIR based on periodically structured metal surfaces. J. Mod. Opt. 2000, 47, 2399-2419.

15. Fleming, J.; Lin, S.; El-Kady, I.; Biswas, R.; Ho, K. All-metallic three-dimensional photonic crystals with a large infrared bandgap. Nature 2002, 417, 52-55.

16. Sai, H.; Kanamori, Y.; Yugami, H. Tuning of the thermal radiation spectrum in the near-infrared region by metallic surface microstructures. J. Micromech. Microeng. 2005, 15, S243.

17. Ghanekar, A.; Sun, M.; Zhang, Z.; Zheng, Y. Optimal Design of Wavelength Selective Thermal Emitter for Thermophotovoltaic Applications. J. Therm. Sci. Eng. Appl. 2007, 10, 011004.

18. Woolf, D.; Hensley, J.; Cederberg, J.; Bethke, D.; Grine, A.; Shaner, E. Heterogeneous metasurface for high temperature selective emission. Appl. Phys. Lett. 2014, 105, 081110.

19. Narayanaswamy, A.; Chen, G. Surface modes for near field thermophotovoltaics. Appl. Phys. Lett. 2003, 82, 3544-3546.

20. DiMatteo, R.; Greiff, P.; Seltzer, D.; Meulenberg, D.; Brown, E.; Carlen, E.; Kaiser, K.; Finberg, S.; Nguyen, H.; Azarkevich, J.; et al. Micron-gap T hermo P hoto V oltaics (MTPV). AIP Conf. Proc. 2004, 738, 42-51.

21. Laroche, M.; Carminati, R.; Greffet, J.J. Near-field thermophotovoltaic energy conversion. J. Appl. Phys. 2006, 100, 063704.

22. Park, K.; Basu, S.; King, W.P.; Zhang, Z. Performance analysis of near-field thermophotovoltaic devices considering absorption distribution. J. Quant. Spectrosc. Radiat. Transf. 2008, 109, 305-316.

23. Francoeur, M.; Vaillon, R.; Mengüç, M.P. Thermal impacts on the performance of nanoscale-gap thermophotovoltaic power generators. IEEE Trans. Energy Convers. 2011, 26, 686-698.

24. Bright, T.; Wang, L.; Zhang, Z. Performance of near-field thermophotovoltaic cells enhanced with a backside reflector. J. Heat Transf. 2014, 136, 062701.

25. Ghanekar, A.; Lin, L.; Su, J.; Sun, H.; Zheng, Y. Role of nanoparticles in wavelength selectivity of multilayered structures in the far-field and near-field regimes. Opt. Express 2015, 23, A1129-A1139.

26. Ghanekar, A.; Lin, L.; Zheng, Y. Novel and efficient Mie-metamaterial thermal emitter for thermophotovoltaic systems. Opt. Express 2016, 24, A868-A877.

27. Francoeur, M.; Basu, S.; Petersen, S.J. Electric and magnetic surface polariton mediated near-field radiative heat transfer between metamaterials made of silicon carbide particles. Opt. Express 2011, 19, 18774-18788.

28. Zhao, Q.; Zhou, J.; Zhang, F.; Lippens, D. Mie resonance-based dielectric metamaterials. Mater. Today 2009, 12, 60-69.

29. Wheeler, M.S.; Aitchison, J.S.; Mojahedi, M. Three-dimensional array of dielectric spheres with an isotropic negative permeability at infrared frequencies. Phys. Rev. B 2005, 72, 193103.

30. Adachi, S. Optical dispersion relations for GaP, GaAs, GaSb, InP, InAs, InSb, $\mathrm{Al}_{x} \mathrm{Ga}_{1-x} \mathrm{As}$, and $\mathrm{In}_{1-x} \mathrm{Ga}_{x} \mathrm{As}_{y} \mathrm{P}_{1-y}$. J. Appl. Phys. 1989, 66, 6030-6040.

31. Ji, T.; Peng, L.; Zhu, Y.; Yang, F.; Cui, Y.; Wu, X.; Liu, L.; He, S.; Zhu, F.; Hao, Y. Plasmonic broadband absorber by stacking multiple metallic nanoparticle layers. Appl. Phys. Lett. 2015, 106, 161107.

32. Sakurai, A.; Kawamata, T. Electromagnetic resonances of solar-selective absorbers with nanoparticle arrays embedded in a dielectric layer. J. Quant. Spectrosc. Radiat. Transf. 2016, 184, 353-359.

33. Reed, J.S. Principles of Ceramics Processing; Wiley: Hoboken, NJ, USA, 1995. 
34. Sahoo, P.K.; Kamal, S.K.; Premkumar, M.; Kumar, T.J.; Sreedhar, B.; Singh, A.; Srivastava, S.; Sekhar, K.C. Synthesis of tungsten nanoparticles by solvothermal decomposition of tungsten hexacarbonyl. Int. J. Refract. Met. Hard Mater. 2009, 27, 784-791.

35. Rodriguez-Suarez, T.; Diaz, L.; Lopez-Esteban, S.; Pecharroman, C.; Esteban-Cubillo, A.; Gremillard, L.; Torrecillas, R.; Moya, J. Epitaxial growth of tungsten nanoparticles on alumina and spinel surfaces. Nanotechnology 2008, 19, 215605.

36. Narayanaswamy, A.; Zheng, Y. A Green's function formalism of energy and momentum transfer in fluctuational electrodynamics. J. Quant. Spectrosc. Radiat. Transf. 2014, 132, 12-21.

37. Chew, W.C. Waves and Fields in Inhomogeneous Media; IEEE Press: New York, NY, USA, 1995.

38. Myroshnychenko, V.; Rodríguez-Fernández, J.; Pastoriza-Santos, I.; Funston, A.M.; Novo, C.; Mulvaney, P.; Liz-Marzán, L.M.; de Abajo, F.J.G. Modelling the optical response of gold nanoparticles. Chem. Soc. Rev. 2008, 37, 1792-1805.

39. Kreibig, U.; Vollmer, M. Optical Properties of Metal Clusters; Springer: Berlin, Germany, 1995; Volume 25.

40. Doyle, W.T. Optical properties of a suspension of metal spheres. Phys. Rev. B 1989, 39, 9852.

41. Wheeler, M.S. A Scattering-Based Approach to the Design, Analysis, and Experimental Verification of Magnetic Metamaterials Made from Dielectrics. Ph.D. Thesis, University of Toronto, Toronto, ON, Canada, 2010.

42. Gao, L.; Lemarchand, F.; Lequime, M. Refractive index determination of SiO2 layer in the UV/Vis/NIR range: Spectrophotometric reverse engineering on single and bi-layer designs. J. Eur. Opt. Soc.-Rapid Publ. 2013, 8, 13010.

43. Rakić, A.D.; Djurišić, A.B.; Elazar, J.M.; Majewski, M.L. Optical properties of metallic films for vertical-cavity optoelectronic devices. Appl. Opt. 1998, 37, 5271-5283.

44. Timans, P. Advances in Rapid Thermal and Integrated Processing; Roozeboom, F., Ed.; Kluwer Academic Publishers: Dordrecht, The Netherlands, 1996.

45. McKelvey, J.P. Solid State and Semiconductor Physics; Harper \& Row: Manhattan, NY, USA, 1966.

(C) 2017 by the authors. Licensee MDPI, Basel, Switzerland. This article is an open access article distributed under the terms and conditions of the Creative Commons Attribution (CC BY) license (http:/ / creativecommons.org/licenses/by/4.0/). 